\title{
Using Multiple Saliencies for the Estimation of Flux, Position, and Velocity in AC Machines
}

\author{
Michael W. Degner and Robert D. Lorenz, Fellow, IEEE
}

\begin{abstract}
This paper presents an improved method of estimating flux angle, rotor position, and velocity by tracking the position of spatial saliencies in an ac machine. Specifically, a machine model is presented which accurately models the behavior of ac machines with multiple spatial harmonic saliencies. The effects of multiple spatial harmonic saliencies on the estimation of flux angle, position, and velocity is analyzed, and methods are presented utilizing multiple spatial harmonic saliencies to provide wide bandwidth, high accuracy estimates of flux angle, rotor position, and velocity.
\end{abstract}

Index Terms - Flux estimation, position estimation, saliency, self sensing, sensorless control.

\section{INTRODUCTION}

$\mathbf{I}$ N RECENT years, "sensorless" or "self-sensing" control of ac machines has been extensively researched. The cost and reliability advantages of eliminating cabling and mechanical sensors for the measurement of position, velocity, and flux have been the driving force behind this research activity.

The techniques used for the estimation of flux, position, and velocity in ac machines can be divided into two groups: 1) tracking speed-dependent phenomenon (back EMF) [1]-[4] and 2) tracking spatial saliencies [5]-[22]. Because the back EMF is a speed-dependent phenomenon, the methods that rely upon it to estimate flux or rotor velocity ultimately fail at low and zero excitation frequency, due to a lack of signal. In addition, because these methods estimate flux or rotor velocity from the voltage and current, they are very sensitive to variations of the machine parameters, especially to the rotor and stator resistances, which vary with temperature. While these approaches tend to work acceptably for medium- to high-speed field orientation and adjustable-speed control, it is the low-speed/low-frequency domain which must be addressed with spatial tracking methods.

The spatial saliency tracking methods can be further subdivided into two groups, depending on whether they use the

Paper IPCSD 98-31, presented at the 1997 Industry Applications Society Annual Meeting, New Orleans, LA, October 5-9, and approved for publication in the IEEE TRANSACTIONS ON INDUSTRY APPLICATIONS by the Industrial Drives Committee of the IEEE Industry Applications Society. This work was supported in part by the Ford Motor Company and in part by the Wisconsin Electric Machines and Power Electronics Consortium (WEMPEC), University of Wisconsin, Madison. Manuscript released for publication April 23, 1998.

M. W. Degner was with the Department of Mechanical Engineering and the Department of Electrical and Computer Engineering, University of Wisconsin, Madison, WI 53706-1572 USA. He is now with Ford Motor Company, Dearborn, MI 48121-2053 USA (e-mail: mdegner@ford.com).

R. D. Lorenz is with the Department of Mechanical Engineering and the Department of Electrical and Computer Engineering, University of Wisconsin, Madison, WI 53706-1572 USA (e-mail: lorenz@engr.wisc.edu).

Publisher Item Identifier S 0093-9994(98)06774-7. fundamental excitation of the machine [5]-[8] or a separate excitation from the fundamental excitation [9]-[22]. Because these methods are tracking a spatial saliency, they are either less sensitive or completely insensitive to parameter variations. The methods that rely upon the fundamental excitation of the machine fail at low and zero speed, due to a lack of signal. The methods that rely upon a separate excitation signal, on the other hand, have no problem with zero- and low-speed operation, because they are not dependent upon the level or frequency of the fundamental excitation. However, the method of separate excitation affects the estimation bandwidth and accuracy that can be achieved.

Some techniques that function at zero speed apply discrete test voltages to the machine, stopping the fundamental excitation during the test cycles [9]-[13]. Due to its discrete nature, an estimation filter/observer is used to provide estimates between test cycles. The limited test cycle time and the filtering effectively trade off noise rejection for estimation bandwidth. An alternative to applying test cycles is to use the inverter bridge to superimpose a continuous polyphase carrier signal (either voltage or current) on the fundamental excitation [14]-[22]. A tracking observer can then be used to continuously track the position of the saliency in the machine, providing a continuous high-bandwidth estimate. This technique utilizes optimal noise rejection techniques taken from image tracking (heterodyning of magnetic, twodimensional (2-D) spatial images) to obtain high-bandwidth, robust flux or rotor position estimates.

A significant limitation to the published methods for zerospeed sensing [9]-[22] is that they have assumed a single sinusoidally distributed spatial saliency is present in the machine. Having more than a single spatial saliency does not prevent any of the methods from working, but it does affect their stability, bandwidth limits, and, most importantly, their estimation accuracy [21]. This paper systematically looks at the effects that multiple spatial saliencies, or harmonics saliencies, have on the estimation of rotor position and flux angle. It presents a model for ac machines with multiple spatial saliencies. Several methods are presented for incorporating this saliency model into the rotor position and flux angle estimate to significantly improve the overall stability, bandwidth, and accuracy of the estimate.

\section{FluX And Rotor Position Estimation for a Single SALIENCY VIA CARRIER SIGNAL INJECTION}

The injection of a carrier signal, in addition to the fundamental excitation, provides a persistent excitation that allows 


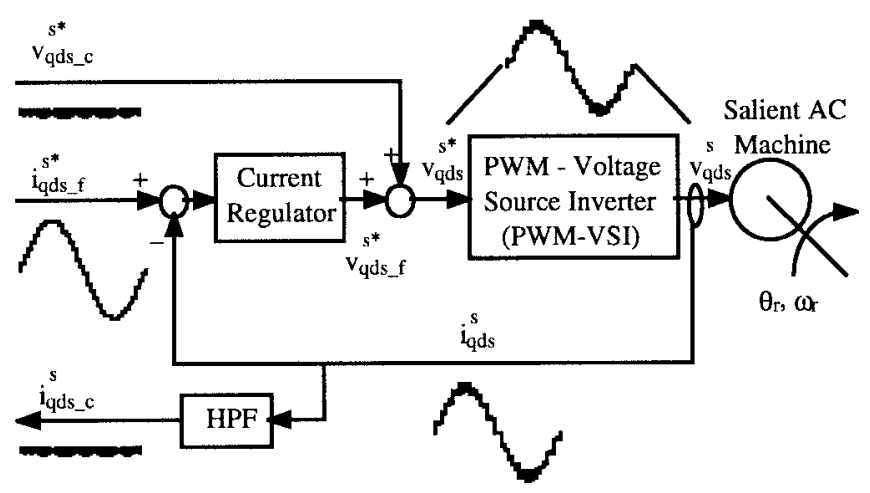

Fig. 1. Injection of carrier signal excitation using a PWM-VSI.

for the continuous estimation of rotor position or flux angle [14]-[22]. One of the simplest forms of carrier signal injection is the injection of a balanced three-phase voltage [14]-[17], [19], [21], [22]. This type of carrier signal injection will produce a carrier signal voltage vector $v_{q d_{-} c}^{s}$ that rotates at the carrier signal frequency $\omega_{c}$ :

$$
v_{q d s_{-} c}^{s}=\left[\begin{array}{c}
v_{q s_{-} c}^{s} \\
v_{d s_{-}}^{s}
\end{array}\right]=V_{s c}\left[\begin{array}{c}
\cos \left(\omega_{c} t\right) \\
-\sin \left(\omega_{c} t\right)
\end{array}\right]=V_{s c} e^{j \omega_{c} t} .
$$

This carrier signal excitation can be injected on top of the fundamental excitation using a pulsewidth modulated voltagesource inverter (PWM-VSI), as shown in Fig. 1.

If the frequency of the carrier signal excitation $\omega_{c}$ is faster than the stator transient time constant $\tau_{\sigma s}$, modeling the machine using only the stator transient inductance $L_{\sigma s}$ is a good approximation:

$$
\begin{aligned}
\tau_{\sigma s} & =\frac{L_{\sigma s}}{r_{s}^{\prime}} \\
v_{q d s_{-} c}^{s} & \cong j \omega_{c} L_{\sigma s} i_{q d s_{-} c}^{s}
\end{aligned}
$$

where

$$
L_{\sigma s}=\left(L_{s}-\frac{L_{m}^{2}}{L_{r}}\right), \quad r_{s}^{\prime}=r_{s}+\left(\frac{L_{m}}{L_{r}}\right)^{2} r_{r}
$$

The stator transient inductance of a machine with a single sinusoidally distributed saliency that has a period equal to the pole pitch can be represented by the following matrix in a reference frame synchronous with the saliency:

$$
L_{\sigma s}^{e}=\left[\begin{array}{cc}
L_{\sigma q s}^{e} & 0 \\
0 & L_{\sigma d s}^{e}
\end{array}\right]
$$

Transforming (4) to the stationary reference frame forms $L_{\sigma s}^{s}=$

$$
\left[\begin{array}{cc}
\sum L_{\sigma s}+\Delta L_{\sigma s} \cos \left(2 \theta_{e}\right) & -\Delta L_{\sigma s} \sin \left(2 \theta_{e}\right) \\
-\Delta L_{\sigma s} \sin \left(2 \theta_{e}\right) & \sum L_{\sigma s}-\Delta L_{\sigma s} \cos \left(2 \theta_{e}\right)
\end{array}\right]
$$

where

$\sum L_{\sigma s}=\frac{1}{2}\left(L_{\sigma q s}^{e}+L_{\sigma d s}^{e}\right)$-average stator transient inductance;

$\Delta L_{\sigma s}=\frac{1}{2}\left(L_{\sigma q s}^{e}-L_{\sigma d s}^{e}\right)$-differential stator transient inductance;

$\theta_{e} \quad$ position of the saliency in electrical degrees.

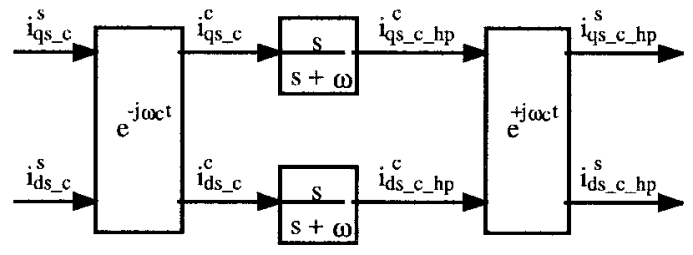

Fig. 2. Synchronous reference frame high-pass filter used to filter off the positive-sequence component of the carrier signal current.

The interaction between the carrier signal voltage vector and the saliency will produce a carrier signal current that contains information relating to the position of the saliency as shown in

$$
\begin{aligned}
i_{q d s_{-} c}^{s} & =\left[\begin{array}{l}
i_{q s_{-} c}^{s} \\
i_{d s_{-} c}^{s}
\end{array}\right]=I_{c_{-} p}\left[\begin{array}{l}
\sin \left(\omega_{c} t\right) \\
\cos \left(\omega_{c} t\right)
\end{array}\right]-I_{c_{-} n}\left[\begin{array}{l}
\sin \left(2 \theta_{e}-\omega_{c} t\right) \\
\cos \left(2 \theta_{e}-\omega_{c} t\right)
\end{array}\right] \\
& =-j I_{c_{-} p} e^{j \omega_{c} t}+j I_{c_{-} n} e^{j\left(2 \theta_{e}-\omega_{c} t\right)}
\end{aligned}
$$

where

$$
I_{c-p}=\left[\frac{\sum L_{\sigma s}}{\sum L_{\sigma s}^{2}-\Delta L_{\sigma s}^{2}}\right] \frac{V_{s c}}{\omega_{c}}
$$

and

$$
I_{c_{-} n}=\left[\frac{\Delta L_{\sigma s}}{\sum L_{\sigma s}^{2}-\Delta L_{\sigma s}^{2}}\right] \frac{V_{s c}}{\omega_{c}} .
$$

The carrier signal current consists of both positive- and negative-sequence components relative to the carrier signal voltage excitation. The positive-sequence component contains no spatial information and is proportional to the average stator transient inductance. The negative-sequence component contains spatial information in its phase and is proportional to the differential stator transient inductance.

Before extracting the spatial information contained in the phase of the negative-sequence carrier signal current, it is desirable to filter off the positive-sequence carrier signal current. Since the positive- and negative-sequence components of the carrier signal current rotate in opposite directions, it is possible to filter off the positive-sequence component by using a highpass filter implemented in a reference frame synchronous with the positive-sequence carrier signal current [22]. Fig. 2 shows a block diagram representing this synchronous reference frame high-pass filter. An equivalent form can also be implemented in the stationary reference frame.

Synchronous reference frame filters (or their stationary reference frame equivalents) can also be used to improve the filtering of the fundamental component of the overall stator current over the filtering that can be achieved with a stationary reference frame high-pass or bandpass filter.

After filtering off the fundamental and positive-sequence carrier signal components of the stator current, the spatial information contained in the phase of the negative-sequence carrier signal current can be extracted using a tracking observer. Fig. 3 shows the block diagram of the tracking observer used to track rotor position when there is a rotor-positiondependent saliency present in the machine $\left(\theta_{e}=\theta_{r}\right)$.

The error signal which drives the tracking observer is formed by taking the vector cross-product between the estimated unit vector of the negative-sequence carrier signal 


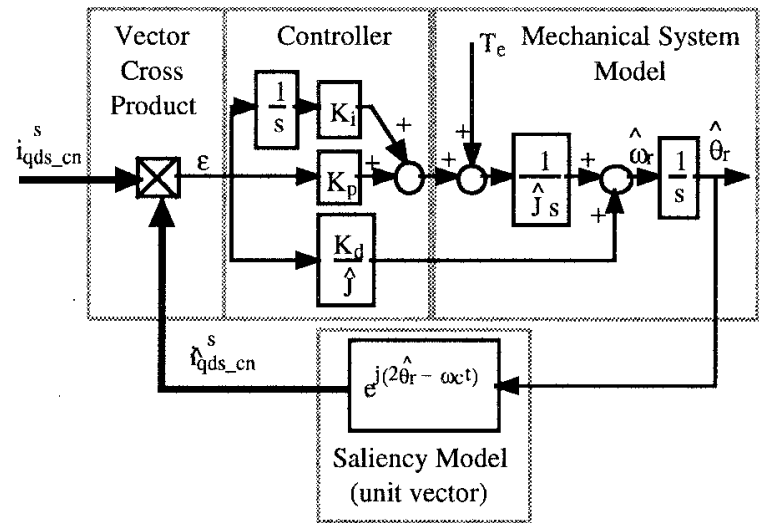

Fig. 3. Tracking observer used to estimate rotor position in a machine with a single rotor-position-dependent saliency.

current vector and the measured negative-sequence carrier signal current vector:

$$
\varepsilon=\hat{i}_{q d s_{-} c n}^{s} \times i_{q d s_{-} c n}^{s}=I_{c_{-} n} \sin \left(2\left(\theta_{r}-\hat{\theta}_{r}\right)\right) .
$$

Because the desired spatial information is present only in the phase of the negative-sequence carrier signal current, the saliency model has to only produce a unit vector estimate of the negative-sequence carrier signal current vector. The magnitudes of the measured and estimated negative-sequence carrier signal current vectors merely scale the phase (tracking) error obtained from the cross-product. Because the estimated negative-sequence carrier signal current vector can be a unit vector, it is independent of any machine parameters.

\section{Multiple SAliEnCIES Machine Model}

The technique shown in Section II assumed that there was a single, sinusoidally distributed saliency, with a period equal to a pole pitch, present in a machine. Although it is possible for a machine to have such a saliency, it is unlikely that this will be the only saliency present in a machine. The discrete nature of the machine windings, nonlinear magnetics, and manufacturing variations will always produce secondary and parasitic saliencies in a machine. Examples of some of these saliencies include stator/rotor slotting, rotor eccentricity, and saturation. Besides being additional saliencies, they also do not necessarily have a period equal to the pole pitch of the machine.

A machine with more than a single sinusoidally distributed saliency, or a nonsinusoidal saliency, can be represented by a Fourier series summation of sinusoidally distributed saliencies. When the machine is excited by a balanced, three-phase, carrier signal voltage excitation, current will be induced, which can be modeled by

$$
i_{q d s_{-} c}^{s}=-j I_{c p} e^{j \omega_{c} t}+j \sum_{i} I_{c n i} e^{j\left(h_{i} \theta_{e i}-\omega_{c} t+\phi_{i}\right)}
$$

where

$I_{c n i}$ magnitude of the $i$ th component of the negative sequence current;

$h_{i} \quad$ harmonic number of the saliency causing the $i$ th component (can be positive, negative, or zero); $\theta_{e i} \quad$ angular position of the saliency causing the $i$ th component;

$\phi_{i} \quad$ phase shift of the $i$ th component relative to the $\theta_{e i}$ reference system.

The magnitude of the individual components of the current can be approximated by

$$
\begin{array}{r}
I_{c p} \cong\left[\frac{\sum L_{\sigma s}}{\sum L_{\sigma s}^{2}-\sum_{j} \Delta L_{\sigma s j}^{2}}\right] \frac{V_{s c}}{\omega_{c}} \\
I_{c n i} \cong\left[\frac{\Delta L_{\sigma s i}}{\sum L_{\sigma s}^{2}-\sum_{j} \Delta L_{\sigma s j}^{2}}\right] \frac{V_{s c}}{\omega_{c}}
\end{array}
$$

where $L_{\sigma s i}$ is the differential stator transient inductance associated with the $i$ th saliency.

As was the case for a single saliency, the resulting carrier signal current consists of both positive- and negative-sequence components. The positive-sequence component contains no spatial information and is proportional to the average stator transient inductance. The negative-sequence components contain the spatial information, with one component caused by each of the saliencies. The magnitudes of the individual negative-sequence components are proportional to the differential stator transient inductance caused by that saliency. It should be noted that the model as presented in (8) does not make a distinction between stationary saliencies $\left(h_{i}=0\right)$, rotor position dependent saliencies $\left(\theta_{e i}=\theta_{r}\right)$, and flux position dependent saliencies $\left(\theta_{e i}=\theta_{r f}, \theta_{e i}=\theta_{s f}\right.$, etc. $)$.

As for the case of a single sinusoidally distributed saliency, the positive-sequence component of the carrier signal current can be filtered off using a synchronous reference frame filter. The interaction of the various saliencies can be seen by transforming the filtered output, i.e., the negative-sequence component of the carrier signal current, to the negativesequence carrier signal synchronous reference frame. Plotting the negative-sequence carrier signal current in polar form results in a pattern that is a "magnetic fingerprint" for that particular machine. From this plot, it is possible to determine the number and type of saliencies present in the machine. Fig. 4 gives some examples of the patterns possible when a machine has two rotating saliencies.

Fig. 4 shows only saliencies that rotate as the rotor or flux in a machine rotate. In addition to a rotating saliency, it is also possible to have a stationary saliency. The effect of a stationary saliency is to shift the shape traced out by the negative-sequence carrier signal current away from the origin, as shown in Fig. 5 for two of the cases originally show in Fig. 4. Although the effect of a stationary saliency may be caused by a true stationary saliency, such as stator winding asymmetry, it can also be caused by unbalanced excitation and scaling differences in the current sensors.

\section{EfFects of Multiple Saliencies on Flux ANGLE AND ROTOR POSITION ESTIMATION}

Estimation errors (reduced accuracy) will result if the technique presented in Section II is used for the estimation of flux angle or rotor position on a machine with multiple saliencies. In general, the components that make up the overall 


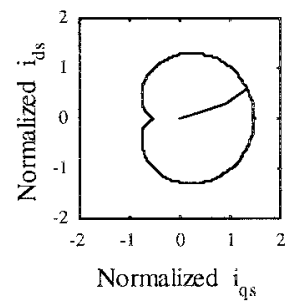

(a)

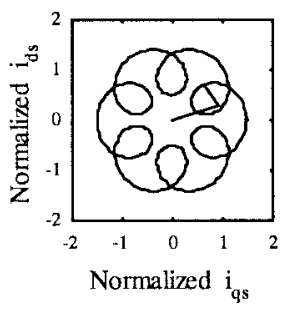

(c)

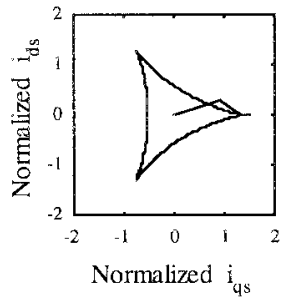

(b)

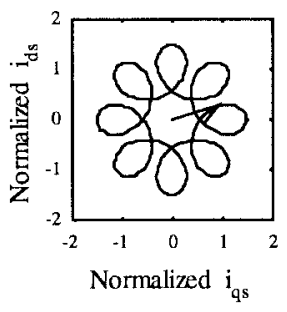

(d)
Fig. 4. Negative-sequence, carrier signal synchronous reference frame polar plot (for a full rotor rotation) of the negative-sequence components of the carrier signal current with various combinations of saliencies. (a) $h_{1}=2, h_{2}=4, I_{c n 2}=0.5 * I_{c n 1}, \phi_{2}=0^{\circ}$. (b) $h_{1}=2, h_{2}=-4, I_{c n 2}=0.5 * I_{c n 1}, \phi_{2}=0^{\circ}$. (c) $h_{1}=2$, $h_{2}=14, I_{c n 2}=0.5 * I_{c n 1}, \phi_{2}=0^{\circ}$. (d) $h_{1}=2$, $h_{2}=-14, I_{c n 2}=0.5 * I_{c n 1}, \phi_{2}=0^{\circ}$.

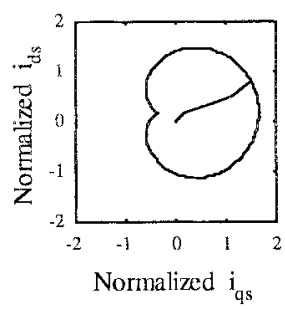

(a)

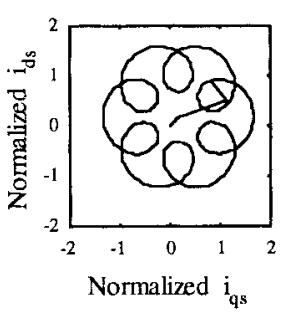

(b)
Fig. 5. Negative-sequence, carrier signal synchronous reference frame polar plot (for a full rotor rotation) of the negative-sequence components of the carrier signal current with a stationary saliency. (a) $h_{1}=2, h_{2}=4, h_{3}=0, I_{c n 2}=0.5 * I_{c n 1}, I_{c n 3}=0.25 * I_{c n 1}$, $\phi_{2}=0^{\circ}, \phi_{3}=45^{\circ}$. (b) $h_{1}=2, h_{2}=14, h_{3}=0, I_{c n 2}=0.5 * I_{c n 1}$, $I_{c n 3}=0.25 * I_{c n 1}, \phi_{2}=0^{\circ}, \phi_{3}=45^{\circ}$.

negative-sequence carrier signal current vector are all in a very narrow frequency range with respect to the observer bandwidth. Because of this, the tracking observer based on a single component will follow the overall negative-sequence carrier signal current vector instead of the modeled component of the negative sequence carrier signal current. This is especially true at low and zero speed, where the components all rotate at approximately the carrier frequency. Fig. 6 shows the rotation of the overall negative-sequence carrier signal current vector in a reference frame synchronous with the component of the negative-sequence carrier signal current that is modeled in the single saliency tracking observer.

The difference in the phase of the overall negative-sequence carrier signal current vector and the modeled component of the negative-sequence carrier signal current will cause

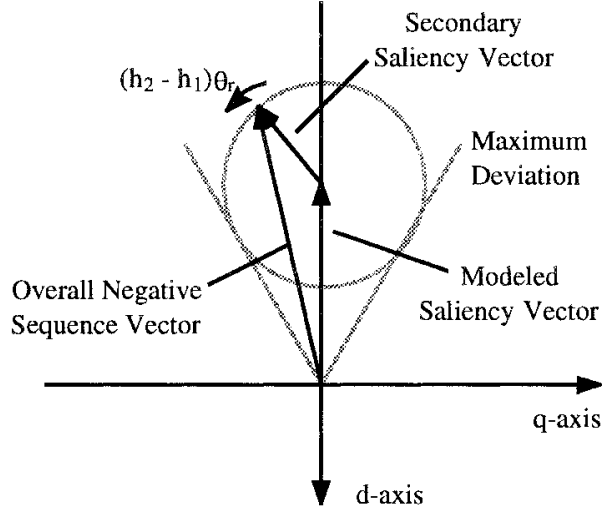

Fig. 6. Rotation of the overall negative-sequence carrier signal current vector in a reference frame synchronous with the modeled component of the negative-sequence carrier signal current.

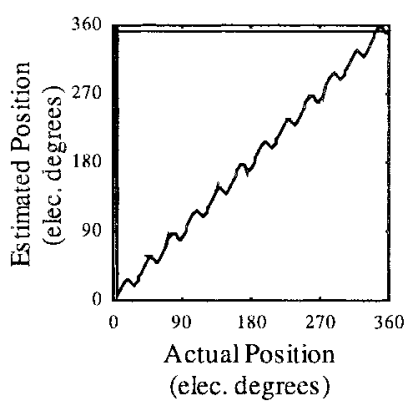

(a)

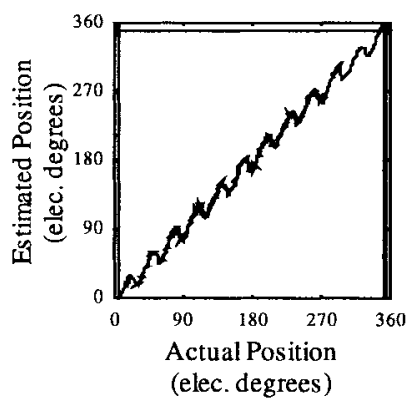

(b)
Fig. 7. Rotor position estimation in a machine with two rotor position dependent saliencies and a tracking observer which only models the primary saliency $h_{1}\left(h_{1}=2, h_{2}=14, I_{c n 2} / I_{c n 1}=0.3125, \phi_{1}=0^{\circ}\right.$, and $\phi_{2}=$ $-10^{\circ}$ ). (a) Theoretical. (b) Experimental.

cyclical errors in rotor or flux position estimates. Fig. 7 shows the theoretical and experimentally measured rotor position estimates as functions of the actual rotor position for a machine with two rotor-position-dependent saliencies when using a tracking observer that only models the primary saliency. Fig. 7 shows that, when multiple saliencies are present, the relationship between the estimated and actual rotor position is no longer the desired, linear, relationship.

The machine used for the estimation in Fig. 7(b) contained a primary saliency $h_{1}$ that was created by deliberately modulating the width of the rotor slot openings. This produced a rotor leakage inductance variation with a period equal to the pole pitch of the machine. The secondary saliency $h_{2}$ was due to the rotor slotting (28 rotor slots). The parameters for the test machine are in Section VI.

A second effect of multiple saliencies, in addition to the estimation error, is to make the dynamics of the tracking observer to be rotor- or flux-position variant. The cause of this variation can be seen in Fig. 6, where the magnitude of the overall negative-sequence carrier signal current vector varies as the saliencies rotate. Since the single saliency tracking observer uses an error signal that is proportional to the magnitude of the signal being tracked, the effective gain of the tracking observer will vary as the saliencies rotate. 


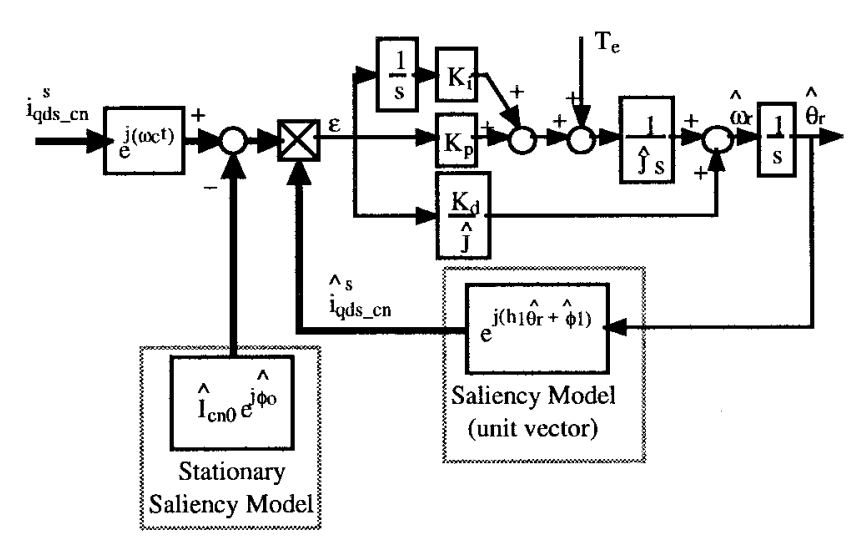

Fig. 8. Tracking observer used to estimate rotor position in a machine with a single rotor-position-dependent saliency and a stationary saliency.

From the plots of estimated rotor position in Fig. 7, it may appear that a simple correction function or lookup table can be used to correct for the errors caused in the flux or position estimation due to multiple saliencies. This can only be done when a unique (single-valued) relationship exists between the estimated and actual positions. When this relationship does not exist (it is not single valued) the estimation error can not be corrected with a lookup table or correction function. Fortunately, the secondary or parasitic saliencies in a machine are often small, allowing the use of a correction function or lookup table to correct for the error.

\section{IMPROVED FLUX AND ROTOR POSITION ESTIMATION InCORPORATING Multiple SALIENCIES MODEL}

By including the knowledge of multiple saliencies in the tracking observer, it is possible to improve the estimation accuracy, even for the case where a correction function or a lookup table is not able to completely improve the estimate. There are several ways that the tracking observer can be modified to improve its estimation accuracy. The following section looks at several of these alternatives.

\section{A. Stationary Saliency Decoupling}

Before looking at the case of multiple rotating saliencies, it is instructive to look at the case where a machine has a single sinusoidally distributed rotating saliency and a stationary saliency. Recalling from Fig. 5, the effect of a stationary saliency was to shift the trajectory of the negative-sequence carrier signal current away from the origin in the negativesequence carrier signal synchronous reference frame. Since the saliency is stationary and its phase and magnitude do not vary with rotor or flux position, the effects of a stationary saliency are easily eliminated by decoupling the component of the negative-sequence carrier signal current vector caused by the stationary saliency. The block diagram of the tracking observer that decouples a stationary saliency is shown in Fig. 8.

Exact decoupling of the stationary saliency is not necessary to dramatically improve the flux or rotor position estimation accuracy and stability. Decoupling does require reasonable knowledge (practical engineering accuracy) of the stationary saliency magnitude and phase. This increases the complexity

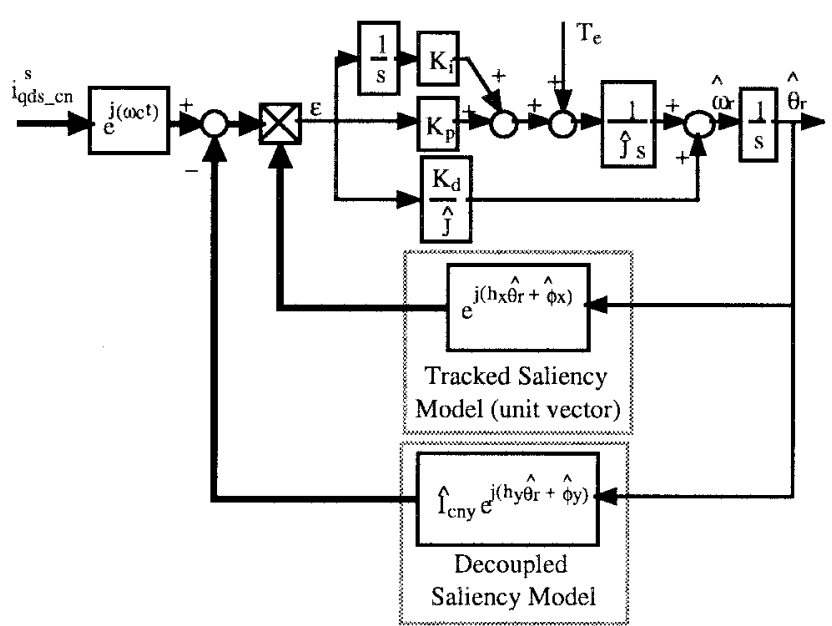

Fig. 9. Decoupling tracking observer used to estimate rotor position in a machine with two rotor-position-dependent saliencies.

and parameter sensitivity of flux or rotor position estimation. Fortunately, stationary saliencies do not typically vary with the machine operating point. The most common sources are not true stationary saliencies, like stator winding asymmetry, but unbalanced excitation, and offsets in the current measurement sensors and A/D converters.

\section{B. Rotating Saliency Decoupling}

The decoupling of the stationary saliency can be extended to the case of a machine with two rotating saliencies, as shown in Fig. 9. Stationary saliency decoupling has been omitted for clarity.

Fig. 9 deliberately does not make a distinction between which of the two saliencies is decoupled and which is tracked. The saliency to decouple can be determined by developing the operating point model for the generic case of decoupling and then choosing the saliency to decouple that guarantees the local stability of the observer. For the tracking observer to be locally stable, the partial derivative of the error term with respect to the estimated rotor position must always be negative (i.e., negative feedback).

Although only shown for the case of two rotating saliencies, the decoupling technique can be extended to cases with additional rotating saliencies. For each additional saliency, another term would have to be decoupled, leaving only a single component of the negative-sequence carrier signal current to be tracked.

An additional benefit of decoupling is the reduction in the operating-point dependency of the tracking observer dynamics. The vector cross-product is now taken between two constant magnitude vectors, resulting in a constant effective gain. Of course, if the decoupling is inexact, some operating-point dependency will remain, but it will be significantly reduced in magnitude when compared with no decoupling.

\section{Multiple Saliency Vector Cross-Product}

Another way of incorporating the knowledge of multiple saliencies in the tracking observer is to improve the 


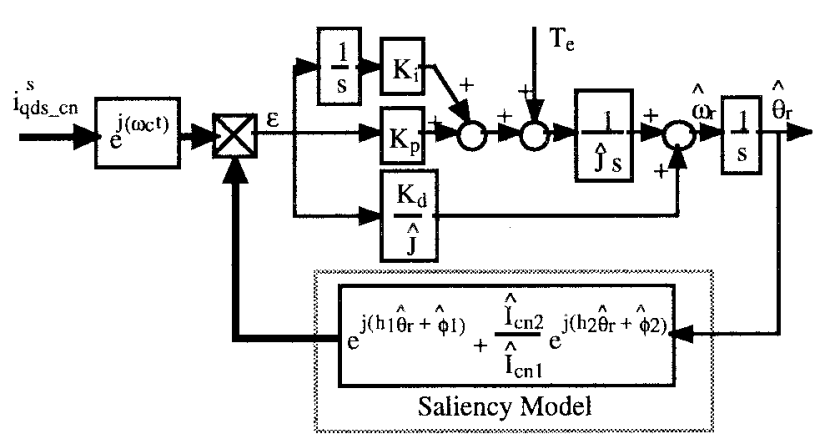

Fig. 10. Multiple saliency tracking observer used to estimate rotor position in a machine with two rotor-position-dependent saliencies.

saliency model, so that it includes all of the significant saliencies present in the machine. The vector cross-product will then be taken between the actual and estimated overall negative-sequence carrier signal current vectors. Fig. 10 shows a tracking observer that models the overall negativesequence carrier signal current vector for the estimation of rotor position in a machine with two rotor-position-dependent saliencies.

Because the saliency model must model the phase of the overall negative-sequence carrier signal current vector correctly, and not its magnitude, the saliency model shown in Fig. 10 is normalized to reduce the number of parameters estimates that are required. The local stability of the tracking observer shown in Fig. 10 can be determined by calculating its operating-point model. This analysis can be used to show that, when the tracking observer in Fig. 10 is locally stable, a correction function or lookup table could also be used to correct for the estimation error caused by the second saliency. Since a lookup table or correction function would, in general, be easier to implement, the tracking observer shown in Fig. 10 is not a very practical solution to the problem.

By adding an offset vector to the overall negative-sequence carrier signal current vector, the observer shown in Fig. 10 can be stabilized over a wider range of saliency ratios. This modified form of the multiple saliency tracking observer is shown in Fig. 11. Operating-point model analysis can be used to determine the necessary magnitude of offset vector to add to stabilize the multiple saliency tracking observer.

Unlike the decoupling form of tracking observer, the dynamics of the multiple saliency tracking observer are operating-point variant. The operating point dependency is caused by the magnitude variation of the overall negativesequence carrier signal current vector. The decoupling form of tracking observer prevents the magnitude variation by decoupling all of the components, except for one. The multiple saliency form of tracking observer, on the other hand, does nothing to counteract the magnitude variations, and its operating point eigenvalues will migrate as a result. Without further modifications, the multiple saliency tracking observer will have inferior dynamics characteristics when compared with the decoupling form of tracking observer.

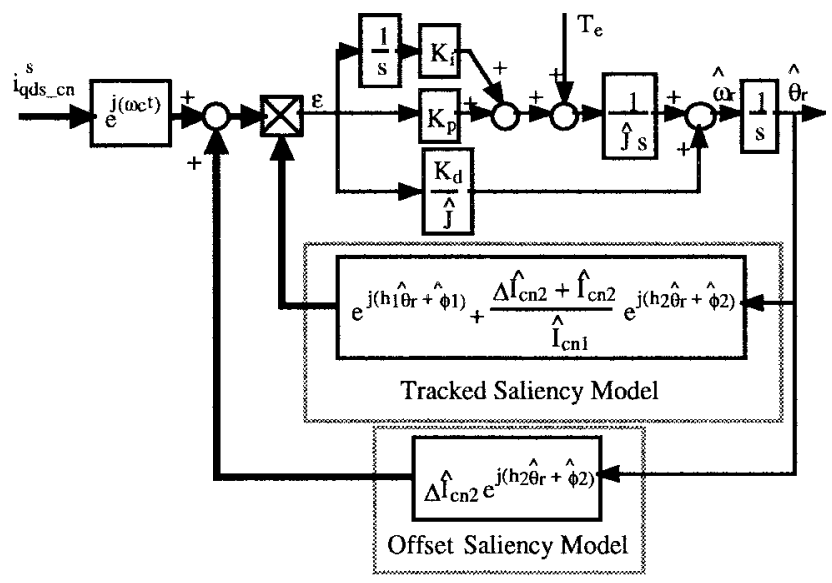

Fig. 11. Modified multiple saliency tracking observer used to estimate rotor position in a machine with two rotor-position-dependent saliencies.

TABLE I

MOTOR PARAMETERS

\begin{tabular}{c|c}
\hline Parameter & Value \\
\hline$r_{s}$ & $0.0135 \Omega$ \\
$r_{r}$ & $0.0140 \Omega$ \\
$L_{l s}$ & $0.065 \mathrm{mH}$ \\
$L_{l r}$ & $0.078 \mathrm{mH}$ \\
$L_{m}$ & $0.530 \mathrm{mH}$ \\
rated speed & $450 \mathrm{r} / \mathrm{min}$ \\
rated torque & $4.82 \mathrm{~N} \cdot \mathrm{m}$ \\
rated current & $120 \mathrm{~A}$ \\
poles & 4 \\
stator slots & 24 \\
rotor slots & 28 \\
dc-bus voltage & $12 \mathrm{~V}$ (automotive) \\
\hline
\end{tabular}

\section{EXPERIMENTAL RESULTS}

The decoupling form of tracking observer was implemented on an induction machine with the parameters shown in Table I, using a Motorola 56000 digital signal processor (DSP).

The machine used for the testing had two rotor-positiondependent saliencies present. The first saliency had a period equal to the pole pitch of the machine $\left(h_{1}=2\right)$ and was created by modifying the rotor slot openings in a sinusoidal pattern with a maximum slot width to minimum slot width ratio of 5 . The second saliency had a period equal to the rotor slot pitch and was caused by the rotor slotting $\left(h_{2}=14\right)$.

A $250-\mathrm{Hz}$ carrier signal rotating voltage vector was injected into the machine. The peak amplitude of the injected carrier signal voltage vector was $15 \%$ of the dc-bus voltage and resulted in a carrier signal current with a peak amplitude of 8.6 A ( $7.2 \%$ of rated). The negative-sequence carrier signal current could be modeled using three saliencies, one stationary and two rotating. The stationary saliency had a component of the negative-sequence carrier signal current with a magnitude of 0.454 A. The likely causes of this stationary saliency were scaling differences in the current measurement sensors, unbalanced excitation, and stator winding asymmetries. The two rotating saliencies resulted in components of the negativesequence carrier signal current that had amplitudes of 0.375 


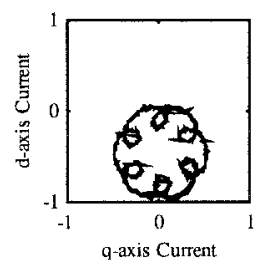

(a)

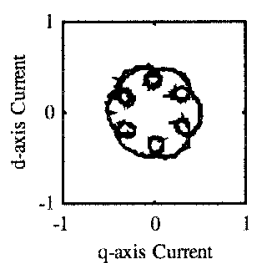

(b)

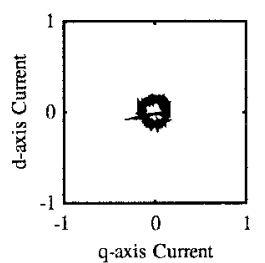

(c)
Fig. 12. Effects of decoupling on the negative-sequence component of the carrier signal current in a negative-sequence carrier signal synchronous reference frame (currents normalized). (a) Overall negative-sequence current (b) Stationary saliency decoupled. (c) Stationary and first rotating saliency decoupled.

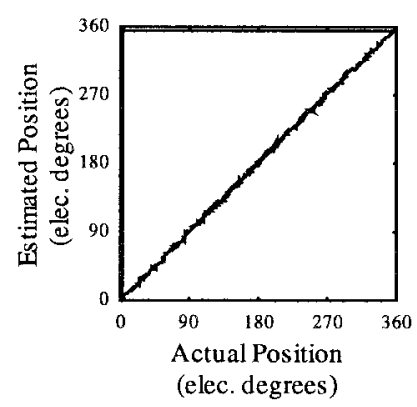

Fig. 13. Rotor position estimation in a machine with two saliencies using a decoupling tracking observer which models both saliencies.

and $0.117 \mathrm{~A}$, respectively. This corresponds to the first saliency having a magnitude equal to $4.4 \%$ of the average stator transient inductance and the second saliency having a magnitude equal to $1.4 \%$ of the average stator transient inductance. Note that the relatively small magnitude of the saliencies present in the machine determined the magnitude of the injected carrier signal voltage. If a larger saliency would have been present, a smaller magnitude carrier signal could have been injected.

Fig. 12 shows the effects of decoupling on the negativesequence component of the carrier signal current in a negativesequence carrier signal synchronous reference frame. Fig. 12(a) shows the overall negative-sequence carrier signal current before any decoupling has been performed. Fig. 12(b) shows the results of decoupling the stationary saliency. Fig. 12(c) shows the results of decoupling the first saliency $h_{1}$. The first, or larger, saliency was decoupled in order to ensure local stability.

With the tracking observer properly tuned, comparison of the estimated and measured rotor position was performed without any fundamental excitation present in the machine. Fig. 13 shows the estimated rotor position as a function of the actual rotor position. Comparison of Fig. 13 with Fig. 7(b) shows the improvement obtained in the rotor position estimate by properly incorporating the knowledge of the major saliencies present in the machine.

Fig. 14 shows a comparison of the estimated and measured rotor position for variations in the rotor speed. For both tests in Fig. 14, the machine started out at zero speed accelerated to a constant negative speed, then to a constant positive speed

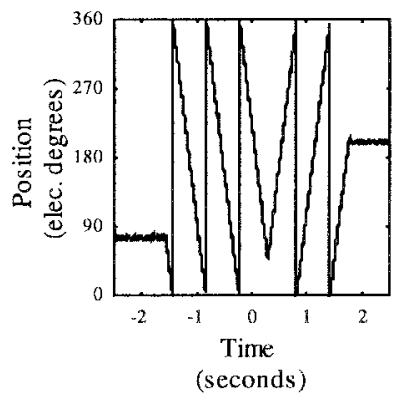

(a)

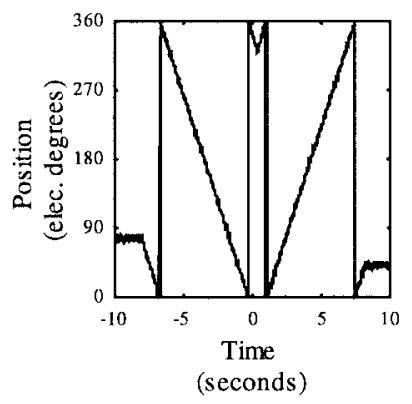

(c)

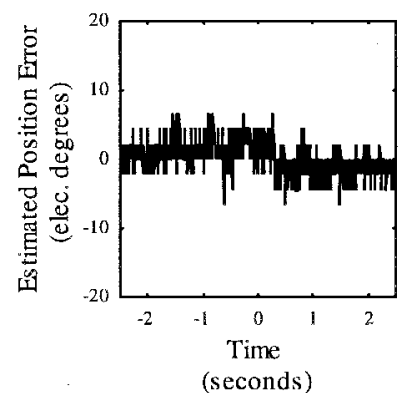

(b)

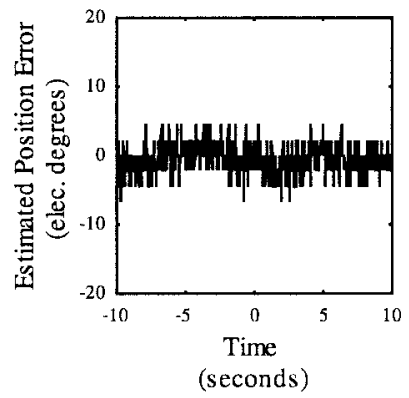

(d)
Fig. 14. Comparison of estimated and measured rotor position. (a) Overlay of estimated and measured rotor position, from 0 to -50 to 50 to $0 \mathrm{r} / \mathrm{min}$. (b) Estimation error, from 0 to -50 to 50 to $0 \mathrm{r} / \mathrm{min}$. (c) Overlay of estimated and measured rotor position, from 0 to -5 to 5 to $0 \mathrm{r} / \mathrm{min}$. (d) Estimation error, from 0 to -5 to 5 to $0 \mathrm{r} / \mathrm{min}$.

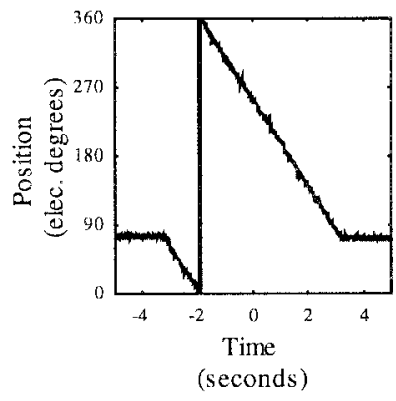

(a)

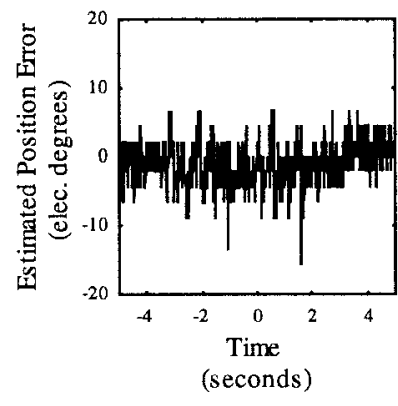

(b)
Fig. 15. Comparison of estimated and measured rotor position for field-oriented test machine producing constant torque equal to $5 \%$ of rated. (a) Overlay of estimated and measured rotor position, from 0 to -5 to 0 $\mathrm{r} / \mathrm{min}$. (b) Estimation error, from 0 to -5 to $0 \mathrm{r} / \mathrm{min}$.

and back to zero speed. From Fig. 14, it is apparent that the rotor position estimate tracks the measured rotor position accurately through speed transients and at zero speed. The 8-b oscilloscope used to capture the data shown in Fig. 14 caused the quantization in the estimated position error plots. Experimental results for higher speeds were not included, since the position estimate does not degrade as the speed increases, and the low speed operation was felt to be most critical and representative of its characteristics.

Fig. 15 shows the estimated and measured rotor position for the machine under indirect field orientation using the estimated rotor position. The test motor produced a constant torque equal 
to $5 \%$ of rated while the dynamometer, which was under speed control, stepped from ) 0 to $-5 \mathrm{r} / \mathrm{min}$ and back to $0 \mathrm{r} / \mathrm{min}$. Again, it is apparent that the estimated rotor position tracks the actual rotor position accurately.

\section{CONCLUSION}

The injection of a carrier signal, in addition to the fundamental excitation, into a machine with known (deterministic) saliencies enables the wide bandwidth estimation of flux angle, rotor position, and velocity.

The use of saliency-based tracking techniques permit highresolution estimation with minimal parameter influence, even in the presence of extreme signal noise.

The persistent excitation provided by the carrier signal excitation allows for continuous estimation, even at sustained low speeds, including zero speed.

The estimation accuracy and robustness of saliency-based techniques are limited by the saliency model used in the estimator. Estimation errors incurred by assuming a single saliency model when multiple saliencies exist have been theoretically and practically demonstrated. In general, estimator accuracy can be significantly improved by properly modeling and incorporating all of the significant saliencies present in the machine.

A basis for modeling machines with multiple saliencies has been presented and verified via experimental work.

Alternative means of tracking the resulting composite vector trajectories have been developed and validated on practical machines.

Although most of the examples given in this paper have been for rotor position (and velocity) estimation, the techniques used are directly applicable to the estimation of flux angle in machines with saturation-induced saliencies.

\section{REFERENCES}

[1] C. Schauder, "Adaptive speed identification for vector control of induction motors without rotational transducers," in Proc. IEEE-IAS Annu. Meeting, San Diego, CA, Oct. 1989, pp. 493-499.

[2] H. Tajima and Y. Hori, "Speed sensorless field-orientation control of the induction machine," IEEE Trans. Ind. Applicat., vol. 29, pp. 175-180, Jan./Feb. 1993.

[3] P. L. Jansen and R. D. Lorenz, "A physically insightful approach to the design and accuracy assessment of flux observers for field oriented induction machine drives," IEEE Trans. Ind. Applicat., vol. 30, pp. 101-110, Jan./Feb. 1994.

[4] P. L. Jansen and R. D. Lorenz, "Accuracy limitations of velocity and flux estimation in direct field oriented induction machines," in Conf. Rec. 4th European Power Electronics (EPE) Conf., Brighton, U.K., Sept. 1993, pp. 312-318.

[5] D. S. Zinger, T. A. Lipo, and D. W. Novotny, "Using induction motor stator windings to extract speed information," in Proc. IEEE-IAS Annu. Meeting, San Diego, CA, Oct. 1989, pp. 213-218.

[6] R. M. Cuzner, R. D. Lorenz, and D. W. Novotny, "Application of nonlinear observers for rotor position detection on an induction motor using machine voltages and currents," in Proc. IEEE-IAS Annu. Meeting, Oct. 1990 , pp. $416-421$.

[7] A. Ferrah, K. G. Bradley, and G. M. Asher, "Sensorless speed detection of inverter fed induction motors using rotor slot harmonics and fast fourier transform," in Proc. PESC'92, 1992, pp. 280-286.

[8] K. J. Binns, D. W. Shimmin, and K. M. Al-Aubidy, "Implicit rotor-position sensing using motor windings for a self-commutating permanent-magnet drive system," Proc. Inst. Elect. Eng., vol. 138, pt. B, no. 1, pp. 28-34, Jan. 1991.

[9] M. Schroedl, "Operation of the permanent magnet synchronous machine without a mechanical sensor," in Proc. IEE Int. Conf. Power Electronics and Variable Speed Drives, London, U.K., July 1990, pp. 51-56.

[10] M. Schroedl, "Sensorless control of induction motors at low speed and standstill," in Proc. ICEM'90, Boston, MA, Aug. 1990, pp. 863-867.

[11] M. Schroedl, D. Hennerbichler, and T. M. Wolbank, "Induction motor drive for electric vehicles without speed- and position sensors," in Conf. Rec. 4th European Power Electronics (EPE) Conf., Brighton, U.K., Sept. 1993, pp. 271-275

[12] M. Schroedl and R. S. Wieser, "Induction motor drive for in locomotives," in Conf. Rec. 5th European Power Electronics (EPE) Conf., Sevilla, Spain, Sept. 19-21, 1995, vol 3., pp. 62-67.

[13] M. Schroedl, "Sensorless control of AC machines at low speed and standstill based on the "INFORM" method," in Proc. IEEE-IAS Annu. Meeting, San Diego, CA, Oct. 5-10, 1996, pp. 270-277.

[14] P. L. Jansen, "The integration of state estimation, control, and design for induction machines," Ph.D. dissertation, Dep. Elect. Comput. Eng., University of Wisconsin, Madison, 1993.

[15] P. L. Jansen and R. D. Lorenz, "Transducerless position and velocity estimation in induction and salient AC machines," IEEE Trans. Ind. Applicat., vol. 31, pp. 240-247, Mar./Apr. 1995.

[16] P. L. Jansen, M. J. Corley, and R. D. Lorenz, "Flux, position, and velocity estimation in $\mathrm{AC}$ machines at zero speed via tracking of high frequency saliencies," in Conf. Rec. 5th European Power Electronics (EPE) Conf., Sevilla, Spain, Sept. 19-21, 1995, vol 3., pp. 154-160.

[17] P. L. Jansen and R. D. Lorenz, "Transducerless field orientation concepts employing saturation-induced saliencies in induction machines," IEEE Trans. Ind. Applicat., vol. 32, pp. 1380-1393, Nov./Dec. 1996.

[18] M. J. Corley and R. D. Lorenz, "Rotor position and velocity estimation for a permanent magnet synchronous machine at standstill and high speeds," in Proc. IEEE-IAS Annu. Meeting, San Diego, CA, Oct. 5-10, 1996, pp. 36-41.

[19] R. D. Lorenz, "Self-sensing methods wide bandwidth position \& velocity sensing at any speed, incl. zero," Tutorial notes for "Sensorless control of AC machines," presented at the IEEE-IAS Annu. Meeting, San Diego, CA, Oct. 5-10, 1996.

[20] F. Blaschke, J. van der Burgt, and A. Vandenput, "Sensorless direct field orientation at zero flux frequency," in Proc. IEEE-IAS Annu. Meeting, San Diego, CA, Oct. 5-10, 1996, pp. 189-196.

[21] J. Cilia, G. M. Asher, and K. Bradley, "Sensorless position detection for vector controlled induction motor drives using an asymmetric outersection cage," in Proc. IEEE-IAS Anпи. Meeting, San Diego, CA, Oct. 5-10, 1996, pp. 286-292.

[22] M. W. Degner and R. D. Lorenz, "Position estimation in induction machines utilizing rotor bar slot harmonics and carrier frequency signal injection," in Proc. Power Conversion Conf., Nagaoka, Japan, Aug. 3-6, 1997, pp. 69-72.

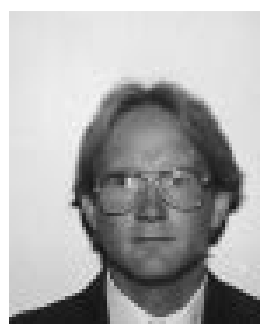

Michael W. Degner received the B.S. degree in 1991, the M.S. degree in 1993, and the Ph.D. degree in 1998, all in mechanical engineering with a focus on electromechanics and control, from the University of Wisconsin, Madison.

$\mathrm{He}$ is currently with Ford Motor Company, Dearborn, MI. His research has concerned the design and electromagnetic modeling of a high pole number, permanent magnet alternator and the estimation of rotor position and flux angle in electric machine drives. His interests include control systems, machine drives, electric machines, power electronics, and electromechanics.

Mr. Degner received the Second Prize Paper Award from the Industrial Drives Committee and the Third Prize Paper Award from the Industrial Power Converter Committee of the IEEE Industry Applications Society at the 1997 Annual Meeting.

Robert D. Lorenz (S'83-M'84-SM'91-F'98), for a photograph and biography, see p. 250 of the March/April 1998 issue of this TRANSACTIONS. 